\title{
Differences in the Antibiotic Resistance Pattern of Staphylococcus aureus Isolated by Clinical Specimens in a University Hospital in South Korea
}

\author{
Seong-No Hong ${ }^{1}$, Joon $\mathrm{Kim}^{2}$, Hyun-Ho Sung ${ }^{1}$ \\ ${ }^{1}$ Department of Clinical Laboratory Science, Dongnam Health University, Suwon, Korea \\ ${ }^{2}$ Department of Laboratory Medicine, Ajou University Hospital, Suwon, Korea
}

\section{일개 대학병원의 임상 검체에서 분리한 포도알균 항생제 내성 경향 차이}

\author{
홍성노 ${ }^{1}$, 김 준 $^{2}$, 성현호 $^{1}$ \\ ${ }^{1}$ 동남보건대학교 임상병리과, ${ }^{2}$ 아주대학교병원 진단검사의학과
}

\begin{abstract}
Information on the prevalence of $S$. aureus and the current antimicrobial resistance profile is necessary in selecting the appropriate treatment of $S$. aureus infections in any part of the world. This study examined the frequency and antibiotic resistance list of $S$. aureus isolates obtained from clinical specimens at one hospital in Korea. A total of 1,746 gram positive cocci collected were identified as $S$. aureus. S. aureus isolates were obtained from different samples including sputum $(\mathrm{N}=565 ; 32.4 \%)$, endotracheal aspirate (358; 20.5\%), wounds (329; 18.8\%), blood $(137 ; 7.8 \%)$, urine $(67 ; 3.8 \%)$, and pus $(59 ; 3.4 \%)$. All 1,545 S. aureus $(100 \%)$ strains screened from sputum (565; 36.6\%), endotracheal aspirate (388; $25.1 \%)$, wounds (329; $21.3 \%)$, blood $(137 ; 8.9 \%)$, urine $(67 ; 4.3 \%)$, and pus $(59 ; 3.8 \%)$ were sensitive to glycopeptide (vancomycin, teicoplanin), oxazolidinone (linezolid) and stretogramin (quinupristin/dalfopristin). The prevalence of resistant $S$. aureus was significantly $(P<0.01)$ lower in urine, blood, pus, wounds, and sputum than in endotracheal aspirates. As a result, there was a significant difference in the antibiotic resistance of $S$. aureus according to the clinical specimens.
\end{abstract}

Key words: Antibiotic resistance, Infection specimen, Staphylococcus aureus

This is an Open Access article distributed under the terms of the Creative Commons Attribution Non-Commercial License (http://creativecommons.org/licenses/by-nc/4.0) which permits unrestricted non-commercial use, distribution, and reproduction in any medium, provided the original work is properly cited.

Copyright (C) 2018 The Korean Society for Clinical Laboratory Science. All rights reserved.
Corresponding author: Hyun-Ho Sung Department of Clinical Laboratory Science, Dongnam Health University, 50

Cheoncheon-ro 74-gil, Jangan-gu, Suwon 16328, Korea

Tel: 82-31-249-6414

Fax: 82-31-249-6410

E-mail:wantyou7@dongnam.ac.kr

Received: May 9, 2018

Revised: May 30, 2018 Accepted: May 31, 2018

\section{INTRODUCTION}

Staphylococcus aureus ( $S$. aureus) is a major human pathogen and gram-positive facultative aerobe that has been found to be the most clinically important species, with broad presence in nature. It is part of the normal flora of human body and commonly carried on the skin or in the nose of healthy individuals, which makes it easy to be transmitted by air or fomites from patients or carriers [1-3]. It been known as one of the most common cause of human infections, such as skin, wound infections and bacteremia. Notwithstanding; the introduction of antibiotics has lowered the mortality rate of $S$. aureus infections. Contrariwise, the bacteria have promptly developed 
resistance mechanisms against many antimicrobial agents [2, 4]. By the mid-1940s, only a few years after its introduction into clinical practice, penicillin resistance was encountered in hospitals and within a decade it had become a significant problem in the community. $S$. aureus is remarkable in its ability to acquire resistance to any antibiotic. Infections caused by antibiotic-resistant strains of $S$. aureus have reached epidemic proportions globally. The overall burden of staphylococcal disease, particularly which caused by methicillin resistant $S$. aureus strains (MRSA), is increasing in many countries in both healthcare and community settings [5]. Methicillin-resistant Staphylococcus aureus (MRSA) has been isolated and recognized more than 50 year ago. MRSA is a specific strain of the $S$. aureus, which is resistant to methicillin and all $\beta$-lactams. Later use of oxacillin as an alternative to methicillin in susceptibility tests resulted in the term 'oxacillin-resistant S. aureus (ORSA) [6], which is resistant to numerous antibiotics. Before the development of antibiotics, invasive infections caused by Staphylococcus aureus have often been fatal [7]. Staphylococcus aureus which is generally susceptible to involvement route in community is mostly MSSA. However, CA-MRSA (community-associated, CA) is recently increased [4, 8]. Antimicrobial resistance is genetically based; resistance is mediated by the acquisition of extra chromosomal genetic elements containing resistance genes. Examples include plasmids, transposable genetic elements, and genomic islands, which are transferred between bacteria through horizontal gene transfer [5]. The mecA gene is a gene having been discovered in bacterial cells which allows a bacterium to be resistant to antibiotics such as methicillin, penicillin and other penicillin-like antibiotics [9]. Borderline resistance, a low-level type of resistance to methicillin exhibited by strains lacking mecA, is associated with modifications in native PBPs, beta-lactamase hyperproduction, or possibly a methicillinase. The resistance phenotype is influenced by numerous factors, including mec and beta-lactamase regulatory elements, fem factors, and yet to be identified chromosomal loci [10]. CA-MRSA strains are increasingly implicated in nosocomial infections, and may eventually displace HA-MRSA strains in hospitals. Consequently, distinctions based on clinical epidemiology and susceptibility are becoming less relevant, arguing in favor of genotypic definitions [11]. MRSA is a bacterium that has been found to be resistant to antibiotics such as methicillin, oxacillin, penicillin and amoxicillin. This organism had become widespread in healthcare settings globally causing different type of infections. Nosocomial infections represent a burden for both patients and the healthcare system because of their association with increased hospitalization costs and high mortality/morbidity rate [12]. The Information of $S$. aureus prevalence and the current antimicrobial resistance profile is necessary in selection of appropriate treatment of $S$. aureus infections in any parts of the world. The present study was carried out to determine the frequency and antibiotic resistance list of $S$. aureusisolates obtained from clinical specimens at a one hospital, Korea.

\section{MATERIALS AND METHODS}

\section{Samples collection}

This study included specimens that were taken from various body sites of infection including blood, wound, sputum, urine, endotracheal aspirate, ear, throat, pleural fluid, peritoneal fluid, cervix and others at Ajou university hospital in Korea from January and May 2016. All clinical specimens were received and collected by clinical laboratory department of assigned hospitals. The identification of isolates was made according to standard methods for any potential clinically significant growth appear on the culture media on the base of quantity, feature of growth, source and site of specimens. The primary identification was made with basic microbiological methods using colony morphology, Gram staining, catalase and coagulase tests. All isolates were confirmed as $S$. aureus by conventional methods including DNase, and mannitol fermentation.

\section{Antibiotic susceptibility test}

The final identification and antibiotic susceptibility 
testing of the bacteria isolated from clinical specimens were obtained using an auto-analyzer system Vitek II system (Biomerieux, Hazelwood, MO, USA). The results of VITIC 60 were confirmed manually using biochemical tests and Kirby-Bauer disk diffusion technique according to CLSI guidelines (M100-27, 2017) [13]. The tested antibiotics included penicillin (PEN), oxacillin (OXA), erythromycin (ERY), gentamicin (GEN), clindamycin (CLI), tetracycline (TET), ciprofloxacin (CIP), fusidic acid (FUS), linezolid (LNZ), vancomycin (VAN), trimethoprimsulfamethoxazole (SXT), cefoxitin (FOX), nitrofurantoin (NIT), levofloxacin (LEV), teicoplanin (TEI) and rifampicin (RIF).

\section{Statistical analysis}

All qualitative variables were analyzed by frequency and expressed as a percentage. The chi-square and Fisher's exact test were used where appropriate using SPSS, PC. version 21.0 (SPSS, Chicago, Illinois, USA). A two-sided $P$ value of $<0.05$ was considered statistically significant.

\section{RESULTS}

\section{Frequency of $S$. aureus isolated from clinical specimens}

A total of 1,746 gram positive cocci that collect in this period identified as $S$. aureus. $S$. aureusisolate belonged to different samples including sputum (N=565; 32.4\%), 358 endotracheal aspirate (20.5\%), 329 wound (18.8\%), 137 blood (7.8\%), 67 urine (3.8\%), 59 pus (3.4\%), 42 ear (2.4\%),
36 throat (2.1\%), 32 peritoneal fluid (1.8\%) 24 catheter (1.4\%), 21 other $(21 ; 1.2 \%)$, cervix $(17 ; 1.0 \%)$ and 59 etc (3.4\%). Frequency of samples collection was shown in Table 1.

\section{Frequency of antibiotic susceptibility by sputum, endotracheal aspirate and wound}

The result on the susceptibility of $S$. aureus of isolates blood, urine and pus was presented in Table 2. Vancomycin, teicoplanin, quinupristin/dalfopristin exhibited the highest activity (100\%). This was followed by erythromycin and clindamycin, ciprofloxacin, fusidic acid. Although ampicillin and penicillin recorded low percentage susceptibilities, isolates were also markedly resistant to methicillin. Higher percentage of intermediate resistance was noted against antibiotics such as erythromycin, erythromycin, clindamycin, fusidic acid, and gentamycin.

\section{Frequency of antibiotic susceptibility by blood, urine and pus}

The highest susceptibility (100\%) occurred in the blood, urine and pus isolates with vancomycin, teicoplanin, trimethoprim/sulfamethoxazole, linezolid while the least occurred with penicillin, oxacillin. Higher percentage of intermediate resistance was noted against antibiotics such as erythromycin, clindamycin, fusidic acid, gentamycin and ciprofloxacin. The susceptibility of $S$. aureus isolates to various antibiotics at blood, urine and pus was presented in Table 3.

Table 1. Frequency of $S$. aureus isolated from clinical specimens $(N=1,746)$

\begin{tabular}{lcllll}
\hline Sample collection & $N(\%)$ & Sample collection & N (\%) & Sample collection & N (\%) \\
\hline Sputum & $565(32.36)$ & Other & $21(1.20)$ & Sinus & $2(0.11)$ \\
Endotracheal aspirate & $358(20.50)$ & Cervix & $17(0.97)$ & BAL fluid & $1(0.06)$ \\
Wound & $329(18.84)$ & Bronx & $14(0.80)$ & CSF & $1(0.06)$ \\
Blood & $137(7.85)$ & Pleural & $10(0.57)$ & Eye DIS & $1(0.06)$ \\
Urine & $67(3.84)$ & Endo tube & $9(0.52)$ & NSSDL AS & $1(0.06)$ \\
Pus & $59(3.38)$ & Bone & $5(0.29)$ & P-D fluid & $1(0.06)$ \\
Ear & $42(2.41)$ & Bile & $4(0.23)$ & Urethra & $1(0.06)$ \\
Throat & $36(2.06)$ & Ascitic fluid & $3(0.17)$ & Vaginal & $1(0.06)$ \\
Peritoneal fluid & $32(1.83)$ & Pancreatic fluid & $3(0.17)$ & & \\
Catheter & $24(1.37)$ & EVD site & $2(0.11)$ & & \\
\hline
\end{tabular}

Abbreviations: EVD, extral ventricular drainage; BAL, bronchoalveolar lavage; Eye DIS, Eye discharge; P-D, peritoneal dialysis. 
Table 2. Frequency of antibiotic susceptibility by sputum, endotracheal aspirate and wound

\begin{tabular}{|c|c|c|c|c|c|c|c|c|c|c|}
\hline \multirow{2}{*}{ Variable } & & \multicolumn{3}{|c|}{ Sputum $N=565$} & \multicolumn{3}{|c|}{ Endotracheal aspirate $\mathrm{N}=388$} & \multicolumn{3}{|c|}{ Wound $N=329$} \\
\hline & & $R$ & I & $\mathrm{S}$ & $R$ & I & $\mathrm{S}$ & $R$ & I & $\mathrm{S}$ \\
\hline \multirow[t]{2}{*}{ Glycopetide } & VAN & $\begin{array}{c}0 \\
(0.0)\end{array}$ & $\begin{array}{c}0 \\
(0.0)\end{array}$ & $\begin{array}{c}565 \\
(100)\end{array}$ & $\begin{array}{c}0 \\
(0.0)\end{array}$ & $\begin{array}{c}0 \\
(0.0)\end{array}$ & $\begin{array}{c}358 \\
(100)\end{array}$ & $\begin{array}{c}0 \\
(0.0)\end{array}$ & $\begin{array}{c}0 \\
(0.0)\end{array}$ & $\begin{array}{c}329 \\
(100)\end{array}$ \\
\hline & TEI & $\begin{array}{c}0 \\
(0.0)\end{array}$ & $\begin{array}{c}0 \\
(0.0)\end{array}$ & $\begin{array}{c}565 \\
(100)\end{array}$ & $\begin{array}{c}0 \\
(0.0)\end{array}$ & $\begin{array}{c}0 \\
(0.0)\end{array}$ & $\begin{array}{c}358 \\
(100)\end{array}$ & $\begin{array}{c}0 \\
(0.0)\end{array}$ & $\begin{array}{c}0 \\
(0.0)\end{array}$ & $\begin{array}{c}329 \\
(100)\end{array}$ \\
\hline Macrolide & ERY & $\begin{array}{c}390 \\
(69.0)\end{array}$ & $\begin{array}{c}0 \\
(0.0)\end{array}$ & $\begin{array}{c}175 \\
(31.0)\end{array}$ & $\begin{array}{c}328 \\
(91.6)\end{array}$ & $\begin{array}{c}0 \\
(0.0)\end{array}$ & $\begin{array}{c}30 \\
(8.4)\end{array}$ & $\begin{array}{c}208 \\
(63.2)\end{array}$ & $\begin{array}{c}0 \\
(0.0)\end{array}$ & $\begin{array}{c}121 \\
(36.8)\end{array}$ \\
\hline Licosamide & CLI & $\begin{array}{c}380 \\
(67.2)\end{array}$ & $\begin{array}{c}0 \\
(0.0)\end{array}$ & $\begin{array}{c}185 \\
(32.8)\end{array}$ & $\begin{array}{c}325 \\
(90.7)\end{array}$ & $\begin{array}{c}0 \\
(0.0)\end{array}$ & $\begin{array}{c}32 \\
(9.3)\end{array}$ & $\begin{array}{c}195 \\
(59.3)\end{array}$ & $\begin{array}{c}0 \\
(0.0)\end{array}$ & $\begin{array}{c}130 \\
(40.7)\end{array}$ \\
\hline Aminoglycoside & GEN & $\begin{array}{c}263 \\
(46.5)\end{array}$ & $\begin{array}{c}8 \\
(1.5)\end{array}$ & $\begin{array}{c}294 \\
(52.0)\end{array}$ & $\begin{array}{c}255 \\
(71.2)\end{array}$ & $\begin{array}{c}3 \\
(0.9)\end{array}$ & $\begin{array}{c}100 \\
(27.9)\end{array}$ & $\begin{array}{c}133 \\
(40.4)\end{array}$ & $\begin{array}{c}15 \\
(4.9)\end{array}$ & $\begin{array}{c}180 \\
(54.7)\end{array}$ \\
\hline Oxazolidinone & LNZ & $\begin{array}{c}0 \\
(0.0)\end{array}$ & $\begin{array}{c}0 \\
(0.0)\end{array}$ & $\begin{array}{c}565 \\
(100)\end{array}$ & $\begin{array}{c}0 \\
(0.0)\end{array}$ & $\begin{array}{c}0 \\
(0.0)\end{array}$ & $\begin{array}{c}358 \\
(100)\end{array}$ & $\begin{array}{c}0 \\
(0.0 \%)\end{array}$ & $\begin{array}{c}0 \\
(0.0)\end{array}$ & $\begin{array}{c}329 \\
(100)\end{array}$ \\
\hline \multirow[t]{2}{*}{ Penicillin } & OXA & $\begin{array}{c}449 \\
(79.5)\end{array}$ & $\begin{array}{c}0 \\
(0.0)\end{array}$ & $\begin{array}{c}116 \\
(20.5)\end{array}$ & $\begin{array}{c}332 \\
(92.7)\end{array}$ & $\begin{array}{c}0 \\
(0.0)\end{array}$ & $\begin{array}{c}26 \\
(7.3)\end{array}$ & $\begin{array}{c}230 \\
(69.9)\end{array}$ & $\begin{array}{c}0 \\
(0.0)\end{array}$ & $\begin{array}{c}99 \\
(30.1)\end{array}$ \\
\hline & PEN & $\begin{array}{c}546 \\
(96.6)\end{array}$ & $\begin{array}{c}0 \\
(0.0)\end{array}$ & $\begin{array}{c}19 \\
(3.4)\end{array}$ & $\begin{array}{c}350 \\
(97.7)\end{array}$ & $\begin{array}{c}0 \\
(0.0)\end{array}$ & $\begin{array}{c}8 \\
(2.3)\end{array}$ & $\begin{array}{c}321 \\
(97.6)\end{array}$ & $\begin{array}{c}0 \\
(0.0)\end{array}$ & $\begin{array}{c}8 \\
(2.4)\end{array}$ \\
\hline Sulfonamide & SXT & $\begin{array}{c}1 \\
(0.2)\end{array}$ & $\begin{array}{c}0 \\
(0.0)\end{array}$ & $\begin{array}{c}564 \\
(99.8)\end{array}$ & $\begin{array}{c}2 \\
(0.6)\end{array}$ & $\begin{array}{c}0 \\
(0.0)\end{array}$ & $\begin{array}{c}356 \\
(99.4)\end{array}$ & $\begin{array}{c}7 \\
(2.2)\end{array}$ & $\begin{array}{c}0 \\
(0.0)\end{array}$ & $\begin{array}{c}322 \\
(97.8)\end{array}$ \\
\hline Streptogramin & SYN & $\begin{array}{c}0 \\
(0.0)\end{array}$ & $\begin{array}{c}0 \\
(0.0)\end{array}$ & $\begin{array}{c}565 \\
(100)\end{array}$ & $\begin{array}{c}0 \\
(0.0)\end{array}$ & $\begin{array}{c}0 \\
(0.0)\end{array}$ & $\begin{array}{c}358 \\
(100)\end{array}$ & $\begin{array}{c}0 \\
(0.0)\end{array}$ & $\begin{array}{c}0 \\
(0.0)\end{array}$ & $\begin{array}{c}329 \\
(100)\end{array}$ \\
\hline Tetracycline & TET & $\begin{array}{c}326 \\
(57.7)\end{array}$ & $\begin{array}{c}0 \\
(0.0)\end{array}$ & $\begin{array}{c}239 \\
(42.3)\end{array}$ & $\begin{array}{c}313 \\
(87.4)\end{array}$ & $\begin{array}{c}0 \\
(0.0)\end{array}$ & $\begin{array}{c}45 \\
(12.6)\end{array}$ & $\begin{array}{c}148 \\
(45.0)\end{array}$ & $\begin{array}{c}0 \\
(0.0)\end{array}$ & $\begin{array}{c}181 \\
(55.0)\end{array}$ \\
\hline Quinolone & CIP & $\begin{array}{c}361 \\
(63.9)\end{array}$ & $\begin{array}{c}10 \\
(1.8)\end{array}$ & $\begin{array}{c}194 \\
(34.3)\end{array}$ & $\begin{array}{c}325 \\
(90.8)\end{array}$ & $\begin{array}{c}0 \\
(0.0)\end{array}$ & $\begin{array}{c}33 \\
(9.2)\end{array}$ & $\begin{array}{c}161 \\
(48.9)\end{array}$ & $\begin{array}{c}0 \\
(0.0)\end{array}$ & $\begin{array}{c}165 \\
(51.1)\end{array}$ \\
\hline Fusidicacid & FUS & $\begin{array}{c}336 \\
(59.5)\end{array}$ & $\begin{array}{c}13 \\
(2.3)\end{array}$ & $\begin{array}{c}216 \\
(38.2)\end{array}$ & $\begin{array}{c}313 \\
(87.4)\end{array}$ & $\begin{array}{c}4 \\
(1.1)\end{array}$ & $\begin{array}{c}41 \\
(11.5)\end{array}$ & $\begin{array}{c}143 \\
(43.5)\end{array}$ & $\begin{array}{c}16 \\
(5.1)\end{array}$ & $\begin{array}{c}169 \\
(51.4)\end{array}$ \\
\hline Rifampicin & RIF & $\begin{array}{c}95 \\
(16.8)\end{array}$ & $\begin{array}{c}9 \\
(1.6)\end{array}$ & $\begin{array}{c}461 \\
(81.6)\end{array}$ & $\begin{array}{c}114 \\
(31.8)\end{array}$ & $\begin{array}{c}0 \\
(0.0)\end{array}$ & $\begin{array}{c}244 \\
(68.2)\end{array}$ & $\begin{array}{c}35 \\
(10.6)\end{array}$ & $\begin{array}{c}0 \\
(0.0)\end{array}$ & $\begin{array}{c}294 \\
(89.4)\end{array}$ \\
\hline
\end{tabular}

Abbreviations: R, Resistance; I, Intermediate; S, Susceptibility; VAN, vancomycin; CLI, clindamycin; ERY, erythromycin; FUS, Fusidic acid; GEN, gentamicin; LNZ, linezolid; OXA, oxacillin; PEN, penicillin; RIF, Rifampicin; SXT, Trimethoprim/Sulfamethoxazole. SYN, quinupristin/dalfopristin; TEI, teicoplanin; TET, tetracycline; CIP, ciprofloxacin.

\section{Difference of antibiotic resistance by specimens}

The results of $S$. aureus resistance type according to status of individuals and source of samples (pus, sputum, wound, urine, blood, endotracheal aspirate) was presented in Table 4 and Figure 1. Resistances were difference in the six kind of specimens except for penicillin, where resistance was seen more especially in isolates from endotracheal aspirate specimen as in isolates from specimens (chi-square test and Fishers exact test, $P<0.05$ ).

\section{DISCUSSION}

In our research we have found that there were mostly differences of antibiotic resistant $S$. aureus strains by clinical specimens. The drug resistance patterns of $S$. aureus isolated from clinical specimens and carrier screening samples were found to be highly variable. All of the examined $S$. aureus strains of the specimens were isolated with pus, sputum, wound, urine, blood, and endotracheal aspirate. In this study, the prevalence and antibiotic susceptibility patterns of various specimen isolates obtained from different clinical and carrier subjects were determined. We isolated 1746 clinical specimens screening samples respectively. All the 28 (1717) region of $S$. aureus strains were obtained from clinical specimens and 1515 (86.7\%) of strains were selected from pus, sputum, wound, urine, blood, and endotracheal aspirate among clinical isolates. In our study, all the 1282 S. aureus $(100 \%)$ strains screened from sputum (565), endotracheal aspirate (388) and wound (329) were sensitive to glycopeptide (vancomycin, teicoplanin), oxazolidinonee (linezolid), oxazolidinone (linezolid) and quinupristin/dalfopristin. However, $565 S$. aureus strains 
Table 3. Frequency of antibiotic susceptibility by blood, urine and pus

N (\%)

\begin{tabular}{|c|c|c|c|c|c|c|c|c|c|c|}
\hline \multirow{2}{*}{ Variable } & & \multicolumn{3}{|c|}{ Blood $N=137$} & \multicolumn{3}{|c|}{ Urine $N=67$} & \multicolumn{3}{|c|}{ Pus $N=59$} \\
\hline & & $R$ & I & S & $R$ & | & $S$ & $R$ & | & $S$ \\
\hline \multirow[t]{2}{*}{ Glycopetide } & VAN & $\begin{array}{c}0 \\
(0.0)\end{array}$ & $\begin{array}{c}0 \\
(0.0)\end{array}$ & $\begin{array}{c}137 \\
(100)\end{array}$ & $\begin{array}{c}0 \\
(0.0)\end{array}$ & $\begin{array}{c}0 \\
(0.0)\end{array}$ & $\begin{array}{c}67 \\
(100)\end{array}$ & $\begin{array}{c}0 \\
(0.0)\end{array}$ & $\begin{array}{c}0 \\
(0.0)\end{array}$ & $\begin{array}{c}59 \\
(100)\end{array}$ \\
\hline & TEI & $\begin{array}{c}0 \\
(0.0)\end{array}$ & $\begin{array}{c}0 \\
(0.0)\end{array}$ & $\begin{array}{c}565 \\
(100)\end{array}$ & $\begin{array}{c}0 \\
(0.0)\end{array}$ & $\begin{array}{c}0 \\
(0.0)\end{array}$ & $\begin{array}{c}358 \\
(100)\end{array}$ & $\begin{array}{c}0 \\
(0.0)\end{array}$ & $\begin{array}{c}0 \\
(0.0)\end{array}$ & $\begin{array}{c}329 \\
(100)\end{array}$ \\
\hline Macrolide & ERY & $\begin{array}{c}68 \\
(49.6)\end{array}$ & $\begin{array}{c}0 \\
(0.0)\end{array}$ & $\begin{array}{c}69 \\
(50.4)\end{array}$ & $\begin{array}{c}26 \\
(38.8)\end{array}$ & $\begin{array}{c}0 \\
(0.0)\end{array}$ & $\begin{array}{c}41 \\
(61.2)\end{array}$ & $\begin{array}{c}27 \\
(45.7)\end{array}$ & $\begin{array}{c}0 \\
(0.0)\end{array}$ & $\begin{array}{c}32 \\
(54.3)\end{array}$ \\
\hline Licosamide & $\mathrm{CLI}$ & $\begin{array}{c}63 \\
(46.0)\end{array}$ & $\begin{array}{c}0 \\
(0.0)\end{array}$ & $\begin{array}{c}74 \\
(54.0)\end{array}$ & $\begin{array}{c}23 \\
(34.3)\end{array}$ & $\begin{array}{c}0 \\
(0.0)\end{array}$ & $\begin{array}{c}44 \\
(65.7)\end{array}$ & $\begin{array}{c}22 \\
(37.2)\end{array}$ & $\begin{array}{c}0 \\
(0.0)\end{array}$ & $\begin{array}{c}37 \\
(62.8)\end{array}$ \\
\hline Aminoglycoside & GEN & $\begin{array}{c}50 \\
(36.5)\end{array}$ & $\begin{array}{c}1 \\
(0.7)\end{array}$ & $\begin{array}{c}86 \\
(62.8)\end{array}$ & $\begin{array}{c}21 \\
(31.3)\end{array}$ & $\begin{array}{c}0 \\
(0.0)\end{array}$ & $\begin{array}{c}46 \\
(68.7)\end{array}$ & $\begin{array}{c}17 \\
(28.8)\end{array}$ & $\begin{array}{c}2 \\
(3.4)\end{array}$ & $\begin{array}{c}40 \\
(67.8)\end{array}$ \\
\hline Oxazolidinone & LNZ & $\begin{array}{c}0 \\
(0.0)\end{array}$ & $\begin{array}{c}0 \\
(0.0)\end{array}$ & $\begin{array}{c}137 \\
(100)\end{array}$ & $\begin{array}{c}0 \\
(0.0)\end{array}$ & $\begin{array}{c}0 \\
(0.0)\end{array}$ & $\begin{array}{c}67 \\
(100)\end{array}$ & $\begin{array}{c}0 \\
(0.0)\end{array}$ & $\begin{array}{c}0 \\
(0.0)\end{array}$ & $\begin{array}{c}59 \\
(100)\end{array}$ \\
\hline \multirow[t]{2}{*}{ Penicillin } & OXA & $\begin{array}{c}86 \\
(63.0)\end{array}$ & $\begin{array}{c}0 \\
(0.0)\end{array}$ & $\begin{array}{c}51 \\
(37.0)\end{array}$ & $\begin{array}{c}49 \\
(73.1)\end{array}$ & $\begin{array}{c}0 \\
(0.0)\end{array}$ & $\begin{array}{c}18 \\
(26.9)\end{array}$ & $\begin{array}{c}36 \\
(61.0)\end{array}$ & $\begin{array}{c}0 \\
(0.0)\end{array}$ & $\begin{array}{c}23 \\
(39.0)\end{array}$ \\
\hline & PEN & $\begin{array}{c}119 \\
(86.9)\end{array}$ & $\begin{array}{c}0 \\
(0.0)\end{array}$ & $\begin{array}{c}18 \\
(13.1)\end{array}$ & $\begin{array}{c}61 \\
(91.0)\end{array}$ & $\begin{array}{c}0 \\
(0.0)\end{array}$ & $\begin{array}{c}6 \\
(9.0)\end{array}$ & $\begin{array}{c}57 \\
(96.6)\end{array}$ & $\begin{array}{c}0 \\
(0.0)\end{array}$ & $\begin{array}{c}2 \\
(3.4)\end{array}$ \\
\hline Sulfonamide & SXT & $\begin{array}{c}0 \\
(0.0)\end{array}$ & $\begin{array}{c}0 \\
(0.0)\end{array}$ & $\begin{array}{c}137 \\
(100)\end{array}$ & $\begin{array}{c}0 \\
(0.0)\end{array}$ & $\begin{array}{c}0 \\
(0.0)\end{array}$ & $\begin{array}{c}67 \\
(100)\end{array}$ & $\begin{array}{c}0 \\
(0.0)\end{array}$ & $\begin{array}{c}0 \\
(0.0)\end{array}$ & $\begin{array}{c}59 \\
(100)\end{array}$ \\
\hline Streptogramin & SYN & $\begin{array}{c}0 \\
(0.0)\end{array}$ & $\begin{array}{c}0 \\
(0.0)\end{array}$ & $\begin{array}{c}137 \\
(100)\end{array}$ & $\begin{array}{c}0 \\
(0.0)\end{array}$ & $\begin{array}{c}0 \\
(0.0)\end{array}$ & $\begin{array}{c}67 \\
(100)\end{array}$ & $\begin{array}{c}0 \\
(0.0)\end{array}$ & $\begin{array}{c}0 \\
(0.0)\end{array}$ & $\begin{array}{c}59 \\
(100)\end{array}$ \\
\hline Tetracycline & TET & $\begin{array}{c}44 \\
(32.1)\end{array}$ & $\begin{array}{c}0 \\
(0.0)\end{array}$ & $\begin{array}{c}93 \\
(67.9)\end{array}$ & $\begin{array}{c}19 \\
(28.3)\end{array}$ & $\begin{array}{c}0 \\
(0.0)\end{array}$ & $\begin{array}{c}48 \\
(71.7)\end{array}$ & $\begin{array}{c}15 \\
(25.4)\end{array}$ & $\begin{array}{c}0 \\
(0.0)\end{array}$ & $\begin{array}{c}44 \\
(74.6)\end{array}$ \\
\hline Quinolone & CIP & $\begin{array}{c}72 \\
(52.5)\end{array}$ & $\begin{array}{c}0 \\
(0.0)\end{array}$ & $\begin{array}{c}65 \\
(47.5)\end{array}$ & $\begin{array}{c}19 \\
(28.4)\end{array}$ & $\begin{array}{c}0 \\
(0.0)\end{array}$ & $\begin{array}{c}48 \\
(71.6)\end{array}$ & $\begin{array}{c}22 \\
(37.3)\end{array}$ & $\begin{array}{c}0 \\
(0.0)\end{array}$ & $\begin{array}{c}37 \\
(62.7)\end{array}$ \\
\hline Fusidic acid & FUS & $\begin{array}{c}53 \\
(38.7)\end{array}$ & $\begin{array}{c}0 \\
(0.0)\end{array}$ & $\begin{array}{c}84 \\
(61.3)\end{array}$ & $\begin{array}{c}12 \\
(17.9)\end{array}$ & $\begin{array}{c}4 \\
(6.0)\end{array}$ & $\begin{array}{c}51 \\
(76.1)\end{array}$ & $\begin{array}{c}24 \\
(40.7)\end{array}$ & $\begin{array}{c}1 \\
(1.7)\end{array}$ & $\begin{array}{c}34 \\
(57.6)\end{array}$ \\
\hline rifampicin & RIF & $\begin{array}{c}13 \\
(9.5)\end{array}$ & $\begin{array}{c}0 \\
(0.0)\end{array}$ & $\begin{array}{c}124 \\
(90.5)\end{array}$ & $\begin{array}{c}1 \\
(1.5)\end{array}$ & $\begin{array}{c}0 \\
(0.0)\end{array}$ & $\begin{array}{c}66 \\
(98.5)\end{array}$ & $\begin{array}{c}1 \\
(1.7)\end{array}$ & $\begin{array}{c}0 \\
(0.0)\end{array}$ & $\begin{array}{c}58 \\
(98.3)\end{array}$ \\
\hline
\end{tabular}

Abbreviation: See Table 2.

Table 4. Difference of antibiotic resistance by specimens

\begin{tabular}{|c|c|c|c|c|c|c|c|c|c|}
\hline $\begin{array}{l}\text { Specimens } \\
\mathrm{N}=1,515\end{array}$ & $\mathrm{CLI}$ & ERY & FUS & GEN & OXA & PEN & RIF & TET & CIP \\
\hline $\begin{array}{l}\text { Pus } \\
59\end{array}$ & $\begin{array}{c}22 \\
(37.2)\end{array}$ & $\begin{array}{c}27 \\
(45.7)\end{array}$ & $\begin{array}{c}24 \\
(40.7)\end{array}$ & $\begin{array}{c}17 \\
(28.8)\end{array}$ & $\begin{array}{c}36 \\
(61.0)\end{array}$ & $\begin{array}{c}57 \\
(96.6)\end{array}$ & $\begin{array}{c}1 \\
(1.7)\end{array}$ & $\begin{array}{c}15 \\
(25.4)\end{array}$ & $\begin{array}{c}22 \\
(37.3)\end{array}$ \\
\hline $\begin{array}{l}\text { Sputum } \\
565\end{array}$ & $\begin{array}{c}380 \\
(67.2)\end{array}$ & $\begin{array}{l}390 \\
(69.0)\end{array}$ & $\begin{array}{c}336 \\
(59.5)\end{array}$ & $\begin{array}{c}263 \\
(46.5)\end{array}$ & $\begin{array}{c}449 \\
(79.5)\end{array}$ & $\begin{array}{c}546 \\
(96.6)\end{array}$ & $\begin{array}{c}95 \\
(16.8)\end{array}$ & $\begin{array}{c}326 \\
(57.7)\end{array}$ & $\begin{array}{c}361 \\
(63.9)\end{array}$ \\
\hline$\chi^{2}$ & $20.9^{\star \star}$ & $13.0^{* \star}$ & $8.3^{*}$ & $7.5^{\star}$ & $10.5^{\text {** }}$ & 0.0 & $10.6^{\star \star}$ & $22.4^{\star \star}$ & $18.8^{\star *}$ \\
\hline $\begin{array}{l}\text { Wound } \\
329\end{array}$ & $\begin{array}{c}195 \\
(59.3)\end{array}$ & $\begin{array}{l}208 \\
(63.2)\end{array}$ & $\begin{array}{l}143 \\
(43.5)\end{array}$ & $\begin{array}{l}133 \\
(40.4)\end{array}$ & $\begin{array}{c}230 \\
(69.9)\end{array}$ & $\begin{array}{c}321 \\
(97.6)\end{array}$ & $\begin{array}{c}35 \\
(10.6)\end{array}$ & $\begin{array}{c}148 \\
(45.0)\end{array}$ & $\begin{array}{c}161 \\
(48.9)\end{array}$ \\
\hline$\chi^{2}$ & $52.0^{\star \star}$ & $33.2^{\star \star}$ & $59.9^{\star}$ & $22.4^{*}$ & $16.8^{\star \star}$ & 7.2 & $33.7^{\star \star}$ & $43.5^{\star \star}$ & $56.7^{\star \star}$ \\
\hline $\begin{array}{l}\text { Urine } \\
67\end{array}$ & $\begin{array}{c}23 \\
(34.3)\end{array}$ & $\begin{array}{c}26 \\
(38.8)\end{array}$ & $\begin{array}{c}12 \\
(17.9)\end{array}$ & $\begin{array}{c}21 \\
(31.3)\end{array}$ & $\begin{array}{c}49 \\
(73.1)\end{array}$ & $\begin{array}{c}61 \\
(91.0)\end{array}$ & $\begin{array}{c}1 \\
(1.5)\end{array}$ & $\begin{array}{c}19 \\
(28.3)\end{array}$ & $\begin{array}{c}19 \\
(28.4)\end{array}$ \\
\hline$\chi^{2}$ & $43.4^{\star *}$ & $33.2^{\star \star}$ & $47.2^{\star \star}$ & $13.8^{\star \star}$ & $11.1^{\text {** }}$ & 5.0 & $22.4^{\star \star}$ & $39.0^{\star \star \star}$ & $48.6^{* *}$ \\
\hline $\begin{array}{l}\text { Blood } \\
137\end{array}$ & $\begin{array}{c}63 \\
(46.0)\end{array}$ & $\begin{array}{c}68 \\
(49.6)\end{array}$ & $\begin{array}{c}53 \\
(38.7)\end{array}$ & $\begin{array}{c}50 \\
(36.5)\end{array}$ & $\begin{array}{c}86 \\
(63.0)\end{array}$ & $\begin{array}{c}119 \\
(86.9)\end{array}$ & $\begin{array}{c}13 \\
(9.5)\end{array}$ & $\begin{array}{c}44 \\
(32.1)\end{array}$ & $\begin{array}{c}72 \\
(52.5)\end{array}$ \\
\hline$\chi^{2}$ & $64.3^{\star \star}$ & $42.8^{\star \star}$ & $74.8^{\star \star}$ & $29.8^{\star \star}$ & $25.3^{\star \star}$ & $31.4^{\star *}$ & $38.3^{\star \star}$ & $58.7^{\star \star}$ & $59.2^{\star \star}$ \\
\hline $\begin{array}{l}\text { Endotracheal aspirate } \\
358\end{array}$ & $\begin{array}{c}325 \\
(90.7)\end{array}$ & $\begin{array}{c}328 \\
(91.6)\end{array}$ & $\begin{array}{c}313 \\
(87.4)\end{array}$ & $\begin{array}{c}255 \\
(71.2)\end{array}$ & $\begin{array}{c}332 \\
(92.7)\end{array}$ & $\begin{array}{c}350 \\
(97.7)\end{array}$ & $\begin{array}{c}114 \\
(31.8)\end{array}$ & $\begin{array}{c}313 \\
(87.4)\end{array}$ & $\begin{array}{c}325 \\
(90.8)\end{array}$ \\
\hline$\chi^{2}$ & $193.9^{\star *}$ & $158.5^{\star * *}$ & $244.5^{\star * *}$ & $127.2^{* *}$ & $87.9^{\star \star}$ & $39.4^{\star *}$ & $110.4^{\star *}$ & $235.9^{* *}$ & $217.1^{\text {** }}$ \\
\hline
\end{tabular}

${ }^{\star} p<0.05,{ }^{*} p<0.01$.

Abbreviation: See Table 2. 


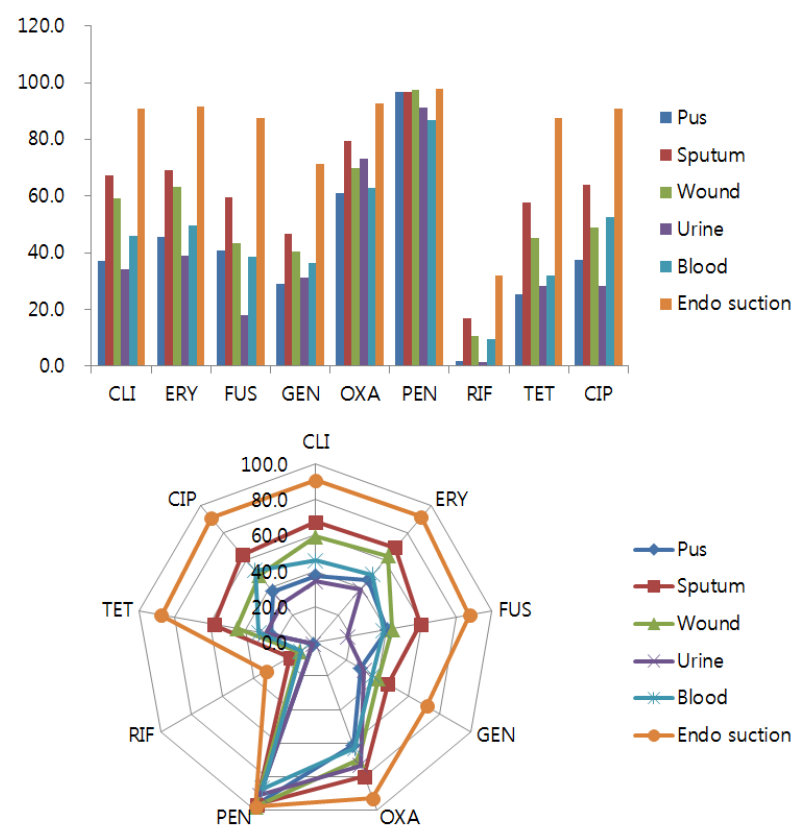

Figure 1. The percentage of antimicrobial resistance profiles of $S$. aureus isolates. Abbreviation: See Table 2.

screened from sputum were resistant to penicillin $(96.9 \%)$, oxacillin (79.5\%), erythromycin (69.0\%), clindamycin (67.2\%), ciprofloxacin (63.9\%), fusidic acid (59.5\%), tetracycline (57.7\%) and aminoglycoside (46.5\%). S. aureus strains screened from endotracheal aspirate recorded resist to penicillin (97.7\%), oxacillin (92.7\%), erythromycin (91.6\%), ciprofloxacin $(90.8 \%)$, clindamycin $(90.7 \%)$, fusidic acid (87.4\%) and tetracycline (87.4\%) which was followed by $71.2 \%$ to gentamycin. S. aureus strains screened from wound recorded resist to penicillin (97.6\%), oxacillin (69.9\%), erythromycin (63.2\%), clindamycin (59.3\%), ciprofloxacin (90.7\%), tetracycline (45.0\%) and fusidic acid (43.5\%) which was followed by $40.4 \%$ to gentamycin. All the 1282 S. aureus (100\%) strains screened from blood (137), urine (67) and pus (59) were sensitive to glycopeptide (vancomycin, teicoplanin), oxazolidinone (linezolid), oxazolidinone (linezolid) and streptogramin (quinupristin/dalfopristin). However, 137 S. aureus strains screened from blood were resistant to penicillin (86.9\%), oxacillin (63.0\%), erythromycin (49.6\%), clindamycin (46.0\%), ciprofloxacin (52.5\%), fusidic acid (38.7\%), tetracycline (32.1\%) and gentamycin (36.5\%). S. aureus strains screened from urine recorded resist to penicillin
(91.0\%), oxacillin (73.1\%), erythromycin (38.8\%), ciprofloxacin (28.4\%), clindamycin (34.3\%), fusidic acid (17.9\%) and tetracycline (28.3\%) which was followed by $31.3 \%$ to gentamycin. S. aureus strains screened from pus recorded resist to penicillin (96.6\%), oxacillin (61.0\%), erythromycin (45.7\%), clindamycin (37.2\%), ciprofloxacin (37.3\%), tetracycline $(24.4 \%)$ and fusidic acid $(40.7 \%)$ which was followed by $28.8 \%$ to gentamycin. Similar results have been reported previously by some authors [14-17]. A total of 830 non-repeated pus/wound swab samples were processed using standard microbiological techniques. Total 76 samples showed the growth of $S$. aureus, among which $36(47.4 \%)$ contained MRSA and $17(22.4 \%)$ samples were found to have $S$. aureus showing inducible clindamycin resistance. Among the $S$. aureus isolated from outpatients, $41.9 \%$ were MRSA. Highest rates of susceptibility of $S$. aureus were seen toward linezolid (100\%) and vancomycin (100\%) [18]. Similar study, a total of 50 specimens of pus, urine, throat swab and sputum were collected to study Staphylococcus aureus. Eight of the isolates showed positive coagulation test and produced characteristic results for Staphylococcus aureus in culture media and biochemical tests. Results of antibiotics sensitivity test revealed the sensitivity of these isolates to penicillin G, oxacillin, erythromycin, gentamycin, amikacin, methicillin, cotrimoxazole, ciprofloxacin, and erythromycin. However, $2 \beta$-hemolytic isolates showed development of spontaneous resistance to $30 \mu \mathrm{g} \mathrm{mL}-1$ of penicillin and $15 \mu \mathrm{g} \mathrm{mL}-1$ of tetracycline [19]. Vancomycin, a glycopeptide in clinical use for more than 50 years, still remains an acceptable treatment option. However, significant concerns have been raised regarding the decreasing susceptibility of $S$. aureus to this agent. The growing awareness of the limitations of vancomycin has served as an impetus for development of newer agents. There has been an increase in the number of agents available to treat MRSA infections. Linezolid, daptomycin, telavancin, and ceftaroline have received regulatory approval in the last decade for the treatment of MRSA [20]. MSSA is common among young patients, traumatic coma, and neurosurgical patients. Most gram-positive organisms 
are sensitive to penicillin, but there is an emergence of methicillin-resistant pathogens which are mostly community acquired [21]. Antimicrobial resistance threatens the effective treatment of bacterial infections and is a serious problem worldwide. Multidrug-resistant bacteria are difficult to treat and the treatment outcome is worse than with susceptible bacteria. In Korea, the antibiotic resistance rates of the major Gram-positive bacteria methicillin-resistant Staphylococcus aureus (MRSA), vancomycin-resistant enterococcus (VRE), and penicillin-resistant Streptococcus pneumonia (PRSP) are very high [22]. MRSA tracheitis is almost exclusively diagnosed in ventilated patients diagnosed with VAT. Underlying risk factors for MRSA respiratory infection include underlying COPD, prior use of antibiotics and multiple hospitalizations. Ventilator Associated Pneumonia (VAP) has high mortality rates and thus, early empirical prescription of broad spectrum antibiotics is mandatory for these patients. Clinicians should be aware of the fact that tracheobronchomalacia can masquerade as asthma in adults and can predispose to the development of serious infections, including MRSA tracheobronchitis in nonventilated adults [23]. The extent of nosocomial $S$. aureus transmission, in particular antibiotic resistant $S$. aureus, the prevalence of $S$. aureus colonization in clinical specimen in hospital was determined. Factors associated with $S$. aureus clinical specimen and antibiotic sensitivity pattern of the isolates were also analyzed. The resistant prevalence of $S$. aureus was significantly lower in urine, blood, pus, wound and sputum as compared to endotracheal aspirate $(P<0.01)$. As a result, there was a difference in antibiotic resistance of $S$. aureus according to the clinical specimens (pus, sputum, wound, urine, blood, endotracheal aspirate). These results are related to the expression of most Staphylococcus aureus virulence factors is controlled by the accessory gene regulator (agr) locus, which encodes a two-component signaling pathway whose activating ligand is an agr-encoded auto inducing peptide (AIP). A polymorphism in the amino acid sequence of the AIP and of its corresponding receptor divides $S$. aureus strains into four major groups. Within a given group, each strain produces a peptide that can activate the agr response in the other member strains, whereas the AIPs belonging to different groups are usually mutually inhibitory [24]. An important property of staphylococci is although typically considered extracellular aerobic pathogens, MRSA also grow and disseminate intracellularly and tolerate anaerobic conditions, all of which are relevant to pulmonary infection. $S$. aureus has evolved into the consummate respiratory pathogen able to not only persist in the respiratory tract but also thrive as an invasive pulmonary pathogen. Strategies to prevent invasive infection will not only need to target the virulence determinants that mediate colonization, iron acquisition, biofilm production, and immune evasion but will also need to be effective against organisms that can disseminate intracellularly [25]. Overall, the most prevalent clinical source of Methicillin-Resistant Staphylococcus aureus (MRSA) isolation belonged to respiratory specimens. MRSA isolates significantly showed higher antibiotic resistance rates compared to methicillin-sensitive Staphylococcus aureus (MSSA) isolates $(p<0.001)$ [26]. Furthermore, such these studies were not much. Therefore, these studies should be continued, and genetic studies of Staphylococcus aureus according to the specimen should be done at the same time.

\section{요 약}

황색포도상구균의 유병률에 대한 정보와 항생제 내성 리스 트는 전 세계 어느 곳에서나 황색포도상구균 감염의 적절한 치 료 방법을 선택하는 데 필요하다. 이 연구는 한국의 어느 병원 임 상 검체에서 얻은 황색포도상구균 균주의 빈도와 항생제 저항 성을 조사하기 위하여 수행하였다. 그람 양성구균 1,746 개의 균은 S. aureus으로 확인되었다. S. aureus은 객담 $(\mathrm{N}=565$, $32.4 \%)$, 기관내 흡인(358, 20.5\%), 상처(329, $18.8 \%)$, 혈액 (137명, $7.8 \%$ ), 고름 $(59 \%, 3.4 \%)$ 이었다. 객담, 기관 내 흡인, 상처, 혈액, 소변 및 고름에서 스크리닝 된 1,282개의 S. aureus 균주 모두 글리코 펩타이드 옥시 졸리 디논(리네 졸리드) 및 스 트렙토그라민(퀴뉴프리스틴/달포프리스틴)에서 모두 민감성 이 나타났다. 황색 포도상 구균은 기관 내 흡인과 비교하면 소변, 혈액, 고름, 상처 및 객담에서 내성이 유의하게 낮았으며 $(P<$ 
0.01), 기관내 흡인 검체에서 분리 된 황색포도상 구균의 경우 특히 내성의 빈도가 높았다. 결과적으로, 임상 표본에 따라 황색 포도상 구균의 항생제 저항성에는 상당한 차이가 있었다.

Acknowledgements: This study was financially supported by Dongnam Health University.

Conflict of interest: None

\section{REFERENCES}

1. Lowy FD. Staphylococcus aureus infections. N Engl J Med. 1998;339:520-532.

2. Hardy KJ, Hawkey PM, Gao F, Oppenheim BA. Methicillin resistant Staphylococcus aureus in the critically ill. Br J Anaesth. 2004;92:121-130.

3. Brown DF, Edwards DI, Hawkey PM, Morrison D, Ridgway GL, Towner KJ, et al. Guidelines for the laboratory diagnosis and susceptibility testing of methicillin-resistant Staphylococcus aureus (MRSA). J Antimicrob Chemother. 2005;56:1000-1018.

4. Palavecino E. Clinical, epidemiological, and laboratory aspects of methicillin-resistant Staphylococcus aureus (MRSA) infections. Methods Mol Biol. 2007;391:1-19.

5. Chambers HF, Deleo FR. Waves of resistance: Staphylococcus aureus in the antibiotic era. Nat Rev Microbiol. 2009;7:629-641.

6. Brown DF, Edwards DI, Hawkey PM, Morrison D, Ridgway GL, Towner KJ, et al. Guidelines for the laboratory diagnosis and susceptibility testing of methicillin-resistant Staphylococcus aureus (MRSA). J Antimicrob Chemother. 2005;56:1000-1018.

7. Joo EJ, Chung DR, Ha YE, Park SY, Kang SJ, Kim SH, et al. Community-associated panton-valentine leukocidin-negative meticillin-resistant Staphylococcus aureus clone (ST72-MRSAIV) causing healthcare-associated pneumonia and surgical site infection in Korea. J Hosp Infect. 2012;81:149-155.

8. Hong SN, Kim J, Sung HH. A study on changes in antimicrobial resistant Staphylococcus aureus from wound isolates in a South Korean university hospital for the past 10 years (2006, 2016). Korean J Clin Lab Sci. 2016;48:335-342.

9. Ubukata K, Nonoguchi R, Matsuhashi M, Konno M. Expression and inducibility in Staphylococcus aureus of the mecA gene, which encodes a methicillin-resistant $S$. aureus-specific penicillin-binding protein. J Bacteriol. 1989;171:2882-2885.

10. Chambers HF. Methicillin resistance in staphylococci: molecular and biochemical basis and clinical implications. Clin Microbiol Rev. 1997;10:781-791.

11. Mediavilla JR, Chen L, Mathema B, Kreiswirth BN. Global epidemiology of community-associated methicillin resistant Staphylococcus aureus (CA-MRSA). Curr Opin Microbiol. 2012;15: 588-595.

12. Al-Zoubi MS, Al-Tayyar IA, Hussein E, Al Jabali A, Khudairat S. Antimicrobial susceptibility pattern of Staphylococcus aureus isolated from clinical specimens in Northern area of Jordan. Iranian journal of microbiology. 2015;7:265-272.

13. Clinical and Laboratory Standards Institute. Performance standards for antimicrobial susceptibility testing; 28th ed, CLSI document M100. Wayne, PA: Clinical and Laboratory Standards Institute; 2017.

14. Akcam FZ, Tinaz GB, Kaya O, Tigli A, Ture E, Hosoglu S. Evaluation of methicillin resistance by cefoxitin disk diffusion and PBP2a latex agglutination test in mecA-positive Staphylococcus aureus, and comparison of mecA with femA, femB, femX positivities. Microbiol Res. 2009;164:400-403.

15. Park C, Seong CN. The correlation between toxin genotype and antibiotic resistance in methicillin resistant Staphylococcus aureus isolated from clinical specimen of intensive care unit. Korean J Clin Lab Sci. 2016;48:202-209.

16. Tamma PD, Robinson GL, Gerber JS, Newland JG, DeLisle CM, Zaoutis TE, et al. Pediatric antimicrobial susceptibility trends across the United States. Infect Control Hosp Epidemiol. 2013;34:1244-1251.

17. Ray GT, Suaya JA, Baxter R. Microbiology of skin and soft tissue infections in the age of community-acquired methicillin-resistant Staphylococcus aureus. Diagn Microbiol Infect Dis. 2013;76:24-30.

18. Belbase A, Pant ND, Nepal K, Neupane B, Baidhya R, Baidya R, et al. Antibiotic resistance and biofilm production among the strains of Staphylococcus aureus isolated from pus/wound swab samples in a tertiary care hospital in Nepal. Ann Clin Microbiol Antimicrob. 2017;16:15. doi: 10.1186/s12941-0170194-0.

19. Begum R, Towhid ST, Moniruzzaman M, Mia Z, Islam MA. Study of Staphylococcus aureus from clinical samples in Savar, Bangladesh. Res J Microbiol. 2011;6:884-890.

20. Choo EJ. Antimicrobial therapy for methicillin-resistant Staphylococcus aureus. J Korean Med Assoc. 2018;61:207-213.

21. Charles MP, Kali A, Easow JM, Joseph NM, Ravishankar M, Srinivasan S, et al. Ventilator-associated pneumonia. Australas Med J. 2014;7:334-344.

22. Jung YH, Kim HB. Multidrug-resistant Gram-positive bacterial infections. Korean J Intern Med. 2015;88:487-501.

23. Kelesidis T, Osman S, Trayner E, Worthington M, Celli B. Tracheobronchitis caused by methicillin-resistant Staphylococcus aureus as a cause of chronic wheezing in a non-ventilated adult patient with tracheobronchomalacia. Respiration. 2010;80: 148-156.

24. Jarraud S, Mougel C, Thioulouse J, Lina G, Meugnier H, Forey F, et al. Relationships between Staphylococcus aureus genetic background, virulence factors, agr groups (alleles), and human disease. Infect Immun. 2002;70:631-641.

25. Parker D, Prince A. Immunopathogenesis of Staphylococcus aureus pulmonary infection. Semin Immunopathol. 2012;34: 281-297.

26. Malekzadegan Y. Motamedifar M. Trends of antibiotic resistance in Staphylococcus aureus isolates obtained from clinical specimens. Jkimsu. 2017;6:19-30. 